\title{
Notes on Binomial Transform of the Generalized Narayana Sequence
}

\author{
Yüksel Soykan \\ Department of Mathematics, Art and Science Faculty, \\ Zonguldak Bülent Ecevit University, 67100, Zonguldak, Turkey \\ e-mail: yuksel_soykan@hotmail.com

\begin{abstract}
In this paper, we define the binomial transform of the generalized Narayana sequence and as special cases, the binomial transform of the Narayana, Narayana-Lucas, Narayana-Perrin sequences will be introduced. We investigate their properties in details.
\end{abstract}

\section{Introduction and Preliminaries}

Recently, there have been so many studies of the sequences of numbers in the literature and the sequences of numbers were widely used in many research areas, such as architecture, nature, art, physics and engineering. The sequence of Fibonacci numbers $\left\{F_{n}\right\}$ is defined by

$$
F_{n}=F_{n-1}+F_{n-2}, \quad n \geq 2, \quad F_{0}=0, F_{1}=1 .
$$

and the sequence of Lucas numbers $\left\{L_{n}\right\}$ is defined by

$$
L_{n}=L_{n-1}+L_{n-2}, \quad n \geq 2, \quad L_{0}=2, L_{1}=1 .
$$

The Fibonacci numbers, Lucas numbers and their generalizations have many interesting properties and applications to almost every field. Horadam 10 defined

Received: April 25, 2021; Accepted: May 28, 2021

2020 Mathematics Subject Classification: 11B39, 11B83.

Keywords and phrases: binomial transform, Narayana sequence, Narayana numbers, binomial transform of Narayana sequence, generalized Tribonacci sequence. 
a generalization of Fibonacci sequence, that is, he defined a second-order linear recurrence sequence $\left\{W_{n}\left(W_{0}, W_{1} ; r, s\right)\right\}$, or simply $\left\{W_{n}\right\}$, as follows:

$$
W_{n}=r W_{n-1}+s W_{n-2} ; \quad W_{0}=a, W_{1}=b, \quad(n \geq 2)
$$

where $W_{0}, W_{1}$ are arbitrary complex numbers and $r, s$ are real numbers, see also Horadam 901112 .

In this paper, we introduce the binomial transform of the generalized Narayana sequence and we investigate, in detail, three special cases which we call them the binomial transform of the Narayana, Narayana-Lucas, Narayana-Perrin sequences. We investigate their properties in the next sections. In this section, we present some properties of the generalized Tribonacci sequence which is a generalization of Fibonacci numbers. The generalized Tribonacci sequence

$$
\left\{W_{n}\left(W_{0}, W_{1}, W_{2} ; r, s, t\right)\right\}_{n \geq 0}
$$

(or shortly $\left\{W_{n}\right\}_{n \geq 0}$ ) is defined as follows:

$$
W_{n}=r W_{n-1}+s W_{n-2}+t W_{n-3}, \quad W_{0}=a, W_{1}=b, W_{2}=c, \quad n \geq 3
$$

where $W_{0}, W_{1}, W_{2}$ are arbitrary complex (or real) numbers and $r, s, t$ are real numbers. This sequence has been studied by many authors, see for example

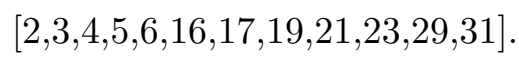

The sequence $\left\{W_{n}\right\}_{n \geq 0}$ can be extended to negative subscripts by defining

$$
W_{-n}=-\frac{s}{t} W_{-(n-1)}-\frac{r}{t} W_{-(n-2)}+\frac{1}{t} W_{-(n-3)}
$$

for $n=1,2,3, \ldots$ when $t \neq 0$. Therefore, recurrence (1.1) holds for all integer $n$.

As $\left\{W_{n}\right\}$ is a third order recurrence sequence (difference equation), it's characteristic equation is

$$
x^{3}-r x^{2}-s x-t=0
$$

whose roots are

$$
\begin{aligned}
& \alpha=\alpha(r, s, t)=\frac{r}{3}+A+M, \\
& \beta=\beta(r, s, t)=\frac{r}{3}+\omega A+\omega^{2} M, \\
& \gamma=\gamma(r, s, t)=\frac{r}{3}+\omega^{2} A+\omega M
\end{aligned}
$$


where

$$
\begin{aligned}
& A=\left(\frac{r^{3}}{27}+\frac{r s}{6}+\frac{t}{2}+\sqrt{\Delta}\right)^{1 / 3}, M=\left(\frac{r^{3}}{27}+\frac{r s}{6}+\frac{t}{2}-\sqrt{\Delta}\right)^{1 / 3}, \\
& \Delta=\Delta(r, s, t)=\frac{r^{3} t}{27}-\frac{r^{2} s^{2}}{108}+\frac{r s t}{6}-\frac{s^{3}}{27}+\frac{t^{2}}{4}, \omega=\frac{-1+i \sqrt{3}}{2}=\exp (2 \pi i / 3) .
\end{aligned}
$$

Note that we have the following identities

$$
\begin{aligned}
\alpha+\beta+\gamma & =r, \\
\alpha \beta+\alpha \gamma+\beta \gamma & =-s, \\
\alpha \beta \gamma & =t .
\end{aligned}
$$

If $\Delta(r, s, t)>0$, then the Equ. (1.2) has one real $(\alpha)$ and two non-real solutions with the latter being conjugate complex. So, in this case, it is well known that the generalized Tribonacci numbers can be expressed, for all integers $n$, using Binet's formula

$$
W_{n}=\frac{p_{1} \alpha^{n}}{(\alpha-\beta)(\alpha-\gamma)}+\frac{p_{2} \beta^{n}}{(\beta-\alpha)(\beta-\gamma)}+\frac{p_{3} \gamma^{n}}{(\gamma-\alpha)(\gamma-\beta)}
$$

where

$$
\begin{gathered}
p_{1}=W_{2}-(\beta+\gamma) W_{1}+\beta \gamma W_{0}, \quad p_{2}=W_{2}-(\alpha+\gamma) W_{1}+\alpha \gamma W_{0}, \\
p_{3}=W_{2}-(\alpha+\beta) W_{1}+\alpha \beta W_{0} .
\end{gathered}
$$

(1.3) can be written in the following form:

$$
W_{n}=M_{1} \alpha^{n}+M_{2} \beta^{n}+M_{3} \gamma^{n}
$$

where

$$
\begin{gathered}
M_{1}=\frac{W_{2}-(\beta+\gamma) W_{1}+\beta \gamma W_{0}}{(\alpha-\beta)(\alpha-\gamma)}, M_{2}=\frac{W_{2}-(\alpha+\gamma) W_{1}+\alpha \gamma W_{0}}{(\beta-\alpha)(\beta-\gamma)}, \\
M_{3}=\frac{W_{2}-(\alpha+\beta) W_{1}+\alpha \beta W_{0}}{(\gamma-\alpha)(\gamma-\beta)} .
\end{gathered}
$$

Note that the Binet form of a sequence satisfying 1.2 for non-negative integers is valid for all integers $n$, for a proof of this result see 13 . This result 
of Howard and Saidak 13 is even true in the case of higher-order recurrence relations.

Next, we give the ordinary generating function $\sum_{n=0}^{\infty} W_{n} x^{n}$ of the sequence $W_{n}$.

Lemma 1.1. Suppose that $f_{W_{n}}(x)=\sum_{n=0}^{\infty} W_{n} x^{n}$ is the ordinary generating function of the generalized Tribonacci sequence $\left\{W_{n}\right\}_{n \geq 0}$. Then, $\sum_{n=0}^{\infty} W_{n} x^{n}$ is given by

$$
\sum_{n=0}^{\infty} W_{n} x^{n}=\frac{W_{0}+\left(W_{1}-r W_{0}\right) x+\left(W_{2}-r W_{1}-s W_{0}\right) x^{2}}{1-r x-s x^{2}-t x^{3}} .
$$

We next find Binet's formula of the generalized Tribonacci sequence $\left\{W_{n}\right\}$ by the use of generating function for $W_{n}$.

Theorem 1.2. (Binet's formula of the generalized Tribonacci numbers) For all integers $n$, we have

$$
W_{n}=\frac{q_{1} \alpha^{n}}{(\alpha-\beta)(\alpha-\gamma)}+\frac{q_{2} \beta^{n}}{(\beta-\alpha)(\beta-\gamma)}+\frac{q_{3} \gamma^{n}}{(\gamma-\alpha)(\gamma-\beta)}
$$

where

$$
\begin{aligned}
& q_{1}=W_{0} \alpha^{2}+\left(W_{1}-r W_{0}\right) \alpha+\left(W_{2}-r W_{1}-s W_{0}\right), \\
& q_{2}=W_{0} \beta^{2}+\left(W_{1}-r W_{0}\right) \beta+\left(W_{2}-r W_{1}-s W_{0}\right), \\
& q_{3}=W_{0} \gamma^{2}+\left(W_{1}-r W_{0}\right) \gamma+\left(W_{2}-r W_{1}-s W_{0}\right) .
\end{aligned}
$$

Note that from 1.3 and 1.5 we have

$$
\begin{aligned}
& W_{2}-(\beta+\gamma) W_{1}+\beta \gamma W_{0}=W_{0} \alpha^{2}+\left(W_{1}-r W_{0}\right) \alpha+\left(W_{2}-r W_{1}-s W_{0}\right), \\
& W_{2}-(\alpha+\gamma) W_{1}+\alpha \gamma W_{0}=W_{0} \beta^{2}+\left(W_{1}-r W_{0}\right) \beta+\left(W_{2}-r W_{1}-s W_{0}\right), \\
& W_{2}-(\alpha+\beta) W_{1}+\alpha \beta W_{0}=W_{0} \gamma^{2}+\left(W_{1}-r W_{0}\right) \gamma+\left(W_{2}-r W_{1}-s W_{0}\right) .
\end{aligned}
$$

In this paper, we consider the case $r=1, s=0, t=1$ and in this case we write $V_{n}=W_{n}$. So, the generalized Narayana sequence $\left\{V_{n}\right\}_{n \geq 0}=\left\{V_{n}\left(V_{0}, V_{1}, V_{2}\right)\right\}_{n \geq 0}$ is defined by the third-order recurrence relations

$$
V_{n}=V_{n-1}+V_{n-3}
$$


with the initial values $V_{0}=c_{0}, V_{1}=c_{1}, V_{2}=c_{2}$ not all being zero.

The sequence $\left\{V_{n}\right\}_{n \geq 0}$ can be extended to negative subscripts by defining

$$
V_{-n}=-V_{-(n-2)}+V_{-(n-3)}
$$

for $n=1,2,3, \ldots$. Therefore, recurrence (1.6) holds for all integer $n$.

1.3 can be used to obtain Binet's formula of generalized Narayana numbers. Binet's formula of generalized Narayana numbers can be given as

$$
V_{n}=\frac{p_{1} \alpha^{n}}{(\alpha-\beta)(\alpha-\gamma)}+\frac{p_{2} \beta^{n}}{(\beta-\alpha)(\beta-\gamma)}+\frac{p_{3} \gamma^{n}}{(\gamma-\alpha)(\gamma-\beta)}
$$

where

$$
\begin{aligned}
& p_{1}=V_{2}-(\beta+\gamma) V_{1}+\beta \gamma V_{0}=V_{0} \alpha^{2}+\left(V_{1}-V_{0}\right) \alpha+\left(V_{2}-V_{1}\right)=q_{1}, \\
& p_{2}=V_{2}-(\alpha+\gamma) V_{1}+\alpha \gamma V_{0}=V_{0} \beta^{2}+\left(V_{1}-V_{0}\right) \beta+\left(V_{2}-V_{1}\right)=q_{2}, \\
& p_{3}=V_{2}-(\alpha+\beta) V_{1}+\alpha \beta V_{0}=V_{0} \gamma^{2}+\left(V_{1}-V_{0}\right) \gamma+\left(V_{2}-V_{1}\right)=q_{3} .
\end{aligned}
$$

Here, $\alpha, \beta$ and $\gamma$ are the roots of the cubic equation $x^{3}-x^{2}-1=0$.

Moreover

$$
\begin{aligned}
& \alpha=\frac{1}{3}+\left(\frac{29}{54}+\sqrt{\frac{31}{108}}\right)^{1 / 3}+\left(\frac{29}{54}-\sqrt{\frac{31}{108}}\right)^{1 / 3} \\
& \beta=\frac{1}{3}+\omega\left(\frac{29}{54}+\sqrt{\frac{31}{108}}\right)^{1 / 3}+\omega^{2}\left(\frac{29}{54}-\sqrt{\frac{31}{108}}\right)^{1 / 3} \\
& \gamma=\frac{1}{3}+\omega^{2}\left(\frac{29}{54}+\sqrt{\frac{31}{108}}\right)^{1 / 3}+\omega\left(\frac{29}{54}-\sqrt{\frac{31}{108}}\right)^{1 / 3}
\end{aligned}
$$

where

$$
\omega=\frac{-1+i \sqrt{3}}{2}=\exp (2 \pi i / 3)
$$

Note that

$$
\begin{aligned}
\alpha+\beta+\gamma & =1, \\
\alpha \beta+\alpha \gamma+\beta \gamma & =0, \\
\alpha \beta \gamma & =1 .
\end{aligned}
$$


Now, we present three special cases of the generalized Narayana sequence $\left\{V_{n}\right\}$. Narayana sequence $\left\{N_{n}\right\}_{n \geq 0}$, Narayana-Lucas sequence $\left\{U_{n}\right\}_{n \geq 0}$, Narayana-Perrin sequence $\left\{H_{n}\right\}_{n \geq 0}$ are defined, respectively, by the third-order recurrence relations

$$
\begin{aligned}
& N_{n+3}=N_{n+2}+N_{n}, \quad N_{0}=0, N_{1}=1, N_{2}=1, \\
& U_{n+3}=U_{n+2}+U_{n}, \quad U_{0}=3, U_{1}=1, U_{2}=1, \\
& H_{n+3}=H_{n+2}+H_{n}, \quad H_{0}=3, H_{1}=0, H_{2}=2 .
\end{aligned}
$$

The sequences $\left\{N_{n}\right\}_{n \geq 0},\left\{U_{n}\right\}_{n \geq 0}$ and $\left\{H_{n}\right\}_{n \geq 0}$ can be extended to negative subscripts by defining

$$
\begin{aligned}
& N_{-n}=-N_{-(n-2)}+N_{-(n-3)} \\
& U_{-n}=-U_{-(n-2)}+U_{-(n-3)} \\
& H_{-n}=-H_{-(n-2)}+H_{-(n-3)}
\end{aligned}
$$

for $n=1,2,3, \ldots$ respectively. Therefore, recurrences (1.7) - 1.9) hold for all integer $n$.

For more details on the generalized Narayana numbers, see Soykan 28. Note that $N_{n}$ is the sequence A000930 in 20] associated with the Narayana's cows sequence and the sequence A078012 in 20 associated with the expansion of (1$x) /\left(1-x-x^{3}\right)$ and $U_{n}$ is the sequence A001609 in 20.

For all integers $n$, Narayana, Narayana-Lucas, Narayana-Perrin numbers (using initial conditions in (1.7)-1.9) can be expressed using Binet's formulas as

$$
\begin{aligned}
N_{n} & =\frac{\alpha^{n+1}}{(\alpha-\beta)(\alpha-\gamma)}+\frac{\beta^{n+1}}{(\beta-\alpha)(\beta-\gamma)}+\frac{\gamma^{n+1}}{(\gamma-\alpha)(\gamma-\beta)}, \\
U_{n} & =\alpha^{n}+\beta^{n}+\gamma^{n}, \\
H_{n} & =\frac{(3+2 \alpha) \alpha^{n-1}}{(\alpha-\beta)(\alpha-\gamma)}+\frac{(3+2 \beta) \beta^{n-1}}{(\beta-\alpha)(\beta-\gamma)}+\frac{(3+2 \gamma) \gamma^{n-1}}{(\gamma-\alpha)(\gamma-\beta)},
\end{aligned}
$$

respectively, see, Soykan 28 for more details.

Next, we give the ordinary generating function $\sum_{n=0}^{\infty} V_{n} x^{n}$ of the generalized Narayana sequence $V_{n}$ (see, Soykan 28 for more details.). 
Lemma 1.3. Suppose that $f_{V_{n}}(x)=\sum_{n=0}^{\infty} V_{n} x^{n}$ is the ordinary generating function of the generalized Narayana sequence $\left\{V_{n}\right\}_{n \geq 0}$. Then, $\sum_{n=0}^{\infty} V_{n} x^{n}$ is given by

$$
\sum_{n=0}^{\infty} V_{n} x^{n}=\frac{V_{0}+\left(V_{1}-V_{0}\right) x+\left(V_{2}-V_{1}\right) x^{2}}{1-x-x^{3}} .
$$

Proof. Take $r=1, s=0, t=1$ in Lemma 1.1 .

The previous lemma gives the following results as particular examples.

Corollary 1.4. Generating functions of Narayana, Narayana-Lucas, Narayana-Perrin numbers are

$$
\begin{aligned}
\sum_{n=0}^{\infty} N_{n} x^{n} & =\frac{x}{1-x-x^{3}}, \\
\sum_{n=0}^{\infty} U_{n} x^{n} & =\frac{3-2 x}{1-x-x^{3}}, \\
\sum_{n=0}^{\infty} H_{n} x^{n} & =\frac{3-3 x+2 x^{2}}{1-x-x^{3}},
\end{aligned}
$$

respectively.

\section{Binomial Transform of the Generalized Narayana Sequence $V_{n}$}

In [15, p. 137], Knuth introduced the idea of the binomial transform. Given a sequence of numbers $\left(a_{n}\right)$, its binomial transform $\left(\hat{a}_{n}\right)$ may be defined by the rule

$$
\hat{a}_{n}=\sum_{i=0}^{n}\left(\begin{array}{c}
n \\
i
\end{array}\right) a_{i}, \quad \text { with inversion } a_{n}=\sum_{i=0}^{n}\left(\begin{array}{c}
n \\
i
\end{array}\right)(-1)^{n-i} \hat{a}_{i},
$$

or, in the symmetric version

$$
\hat{a}_{n}=\sum_{i=0}^{n}\left(\begin{array}{c}
n \\
i
\end{array}\right)(-1)^{i+1} a_{i}, \text { with inversion } a_{n}=\sum_{i=0}^{n}\left(\begin{array}{c}
n \\
i
\end{array}\right)(-1)^{i+1} \hat{a}_{i} .
$$


For more information on binomial transform, see, for example, 7,81830 and references therein.

In this section, we define the binomial transform of the generalized Narayana sequence $V_{n}$ and as special cases the binomial transform of the Narayana, Narayana-Lucas, Narayana-Perrin sequences will be introduced.

Definition 2.1. The binomial transform of the generalized Narayana sequence $V_{n}$ is defined by

$$
b_{n}=\widehat{V}_{n}=\sum_{i=0}^{n}\left(\begin{array}{l}
n \\
i
\end{array}\right) V_{i} .
$$

The few terms of $b_{n}$ are

$$
\begin{aligned}
& b_{0}=\sum_{i=0}^{0}\left(\begin{array}{l}
0 \\
i
\end{array}\right) V_{i}=V_{0}, \\
& b_{1}=\sum_{i=0}^{1}\left(\begin{array}{l}
1 \\
i
\end{array}\right) V_{i}=V_{0}+V_{1}, \\
& b_{2}=\sum_{i=0}^{2}\left(\begin{array}{l}
2 \\
i
\end{array}\right) V_{i}=V_{0}+2 V_{1}+V_{2} .
\end{aligned}
$$

Translated to matrix language, $b_{n}$ has the nice (lower-triangular matrix) form

$$
\left(\begin{array}{c}
b_{0} \\
b_{1} \\
b_{2} \\
b_{3} \\
b_{4} \\
\vdots
\end{array}\right)=\left(\begin{array}{cccccc}
1 & 0 & 0 & 0 & 0 & \cdots \\
1 & 1 & 0 & 0 & 0 & \cdots \\
1 & 2 & 1 & 0 & 0 & \cdots \\
1 & 3 & 3 & 1 & 0 & \cdots \\
1 & 4 & 6 & 4 & 1 & \cdots \\
\vdots & \vdots & \vdots & \vdots & \vdots & \ddots
\end{array}\right)\left(\begin{array}{c}
V_{0} \\
V_{1} \\
V_{2} \\
V_{3} \\
V_{4} \\
\vdots
\end{array}\right) .
$$

As special cases of $b_{n}=\widehat{V}_{n}$, the binomial transforms of the Narayana, Narayana-Lucas, Narayana-Perrin sequences are defined as follows: The binomial transform of the Narayana sequence $N_{n}$ is

$$
\widehat{N}_{n}=\sum_{i=0}^{n}\left(\begin{array}{l}
n \\
i
\end{array}\right) N_{i},
$$


the binomial transform of the Narayana-Lucas sequence $U_{n}$ is

$$
\widehat{U}_{n}=\sum_{i=0}^{n}\left(\begin{array}{l}
n \\
i
\end{array}\right) U_{i},
$$

the binomial transform of the Narayana-Perrin sequence $H_{n}$ is

$$
\widehat{H}_{n}=\sum_{i=0}^{n}\left(\begin{array}{l}
n \\
i
\end{array}\right) H_{i} .
$$

Lemma 2.2. For $n \geq 0$, the binomial transform of the generalized Narayana sequence $V_{n}$ satisfies the following relation:

$$
b_{n+1}=\sum_{i=0}^{n}\left(\begin{array}{c}
n \\
i
\end{array}\right)\left(V_{i}+V_{i+1}\right) .
$$

Proof. We use the following well-known identity:

$$
\left(\begin{array}{c}
n+1 \\
i
\end{array}\right)=\left(\begin{array}{c}
n \\
i
\end{array}\right)+\left(\begin{array}{c}
n \\
i-1
\end{array}\right)
$$

Note also that

$$
\left(\begin{array}{c}
n+1 \\
0
\end{array}\right)=\left(\begin{array}{c}
n \\
0
\end{array}\right)=1 \text { and }\left(\begin{array}{c}
n \\
n+1
\end{array}\right)=0
$$

Then

$$
\begin{aligned}
b_{n+1} & =V_{0}+\sum_{i=1}^{n+1}\left(\begin{array}{c}
n+1 \\
i
\end{array}\right) V_{i} \\
& =V_{0}+\sum_{i=1}^{n+1}\left(\begin{array}{c}
n \\
i
\end{array}\right) V_{i}+\sum_{i=1}^{n+1}\left(\begin{array}{c}
n \\
i-1
\end{array}\right) V_{i} \\
& =V_{0}+\sum_{i=1}^{n}\left(\begin{array}{c}
n \\
i
\end{array}\right) V_{i}+\sum_{i=0}^{n}\left(\begin{array}{c}
n \\
i
\end{array}\right) V_{i+1} \\
& =\sum_{i=0}^{n}\left(\begin{array}{c}
n \\
i
\end{array}\right) V_{i}+\sum_{i=0}^{n}\left(\begin{array}{c}
n \\
i
\end{array}\right) V_{i+1} \\
& =\sum_{i=0}^{n}\left(\begin{array}{c}
n \\
i
\end{array}\right)\left(V_{i}+V_{i+1}\right) .
\end{aligned}
$$

This completes the proof. 
Remark 2.3. From the last Lemma, we see that

$$
b_{n+1}=b_{n}+\sum_{i=0}^{n}\left(\begin{array}{l}
n \\
i
\end{array}\right) V_{i+1} .
$$

The following theorem gives recurrent relations of the binomial transform of the generalized Narayana sequence.

Theorem 2.4. For $n \geq 0$, the binomial transform of the generalized Narayana sequence $V_{n}$ satisfies the following recurrence relation:

$$
b_{n+3}=4 b_{n+2}-5 b_{n+1}+3 b_{n} .
$$

Proof. To show (2.1), by writing

$$
b_{n+3}=r_{1} \times b_{n+2}+s_{1} \times b_{n+1}+t_{1} \times b_{n}
$$

and taking the values $n=0,1,2$ and then solving the system of equations

$$
\begin{aligned}
& b_{3}=r_{1} \times b_{2}+s_{1} \times b_{1}+t_{1} \times b_{0} \\
& b_{4}=r_{1} \times b_{3}+s_{1} \times b_{2}+t_{1} \times b_{1} \\
& b_{5}=r_{1} \times b_{4}+s_{1} \times b_{3}+t_{1} \times b_{2}
\end{aligned}
$$

we find that $r_{1}=4, s_{1}=-5, t_{1}=3$.

The sequence $\left\{b_{n}\right\}_{n \geq 0}$ can be extended to negative subscripts by defining

$$
b_{-n}=\frac{5}{3} b_{-n+1}-\frac{4}{3} b_{-n+2}+\frac{1}{3} b_{-n+3}
$$

for $n=1,2,3, \ldots$. Therefore, recurrence (2.1) holds for all integer $n$.

Note that the recurence relation (2.1) is independent from initial values. So,

$$
\begin{aligned}
\widehat{N}_{n+3} & =4 \widehat{N}_{n+2}-5 \widehat{N}_{n+1}+3 \widehat{N}_{n}, \\
\widehat{U}_{n+3} & =4 \widehat{U}_{n+2}-5 \widehat{U}_{n+1}+3 \widehat{U}_{n}, \\
\widehat{H}_{n+3} & =4 \widehat{H}_{n+2}-5 \widehat{H}_{n+1}+3 \widehat{H}_{n} .
\end{aligned}
$$


and

$$
\begin{aligned}
\widehat{N}_{-n} & =\frac{5}{3} \widehat{N}_{-n+1}-\frac{4}{3} \widehat{N}_{-n+2}+\frac{1}{3} \widehat{N}_{-n+3}, \\
\widehat{U}_{-n} & =\frac{5}{3} \widehat{U}_{-n+1}-\frac{4}{3} \widehat{U}_{-n+2}+\frac{1}{3} \widehat{U}_{-n+3}, \\
\widehat{H}_{-n} & =\frac{5}{3} \widehat{H}_{-n+1}-\frac{4}{3} \widehat{H}_{-n+2}+\frac{1}{3} \widehat{H}_{-n+3} .
\end{aligned}
$$

The first few terms of the binomial transform of the generalized Narayana sequence with positive subscript and negative subscript are given in the following Table 1.

Table 1: A few binomial transform (terms) of the generalized Narayana sequence.

\begin{tabular}{ccc}
\hline$n$ & $b_{n}$ & $b_{-n}$ \\
\hline 0 & $V_{0}$ & $\frac{1}{3}\left(2 V_{0}-2 V_{1}+V_{2}\right)$ \\
1 & $V_{0}+V_{1}$ & $\frac{1}{9}\left(V_{0}-7 V_{1}+5 V_{2}\right)$ \\
2 & $V_{0}+2 V_{1}+V_{2}$ & $-\frac{1}{27}\left(10 V_{0}+11 V_{1}-13 V_{2}\right)$ \\
3 & $2 V_{0}+3 V_{1}+4 V_{2}$ & $-\frac{1}{81}\left(44 V_{0}-11 V_{1}-14 V_{2}\right)$ \\
4 & $6 V_{0}+5 V_{1}+11 V_{2}$ & $-\frac{1}{243}\left(91 V_{0}-124 V_{1}+41 V_{2}\right)$ \\
5 & $17 V_{0}+11 V_{1}+27 V_{2}$ & $-\frac{1}{729}\left(17 V_{0}-389 V_{1}+256 V_{2}\right)$ \\
6 & $44 V_{0}+28 V_{1}+65 V_{2}$ & $\frac{1}{2187}\left(611 V_{0}+556 V_{1}-662 V_{2}\right)$ \\
7 & $109 V_{0}+72 V_{1}+158 V_{2}$ & $\frac{1}{6561}\left(2440 V_{0}-772 V_{1}-607 V_{2}\right)$ \\
8 & $267 V_{0}+181 V_{1}+388 V_{2}$ & $\frac{1}{19683}\left(4715 V_{0}-7031 V_{1}+2605 V_{2}\right)$ \\
9 & $655 V_{0}+448 V_{1}+957 V_{2}$ & $\frac{1}{59049}\left(206 V_{0}+20887 V_{1}-14351 V_{2}\right)$ \\
10 & $1612 V_{0}+1103 V_{1}+2362 V_{2}$ & $-\frac{1}{177147}\left(35650 V_{0}+27011 V_{1}-35032 V_{2}\right)$ \\
11 & $3974 V_{0}+2715 V_{1}+5827 V_{2}$ & $\frac{1}{531441}\left(133343 V_{0}-52310 V_{1}-26393 V_{2}\right)$ \\
12 & $9801 V_{0}+6689 V_{1}+14369 V_{2}$ & $-\frac{1}{1594323}\left(240769 V_{0}-397699 V_{1}+159260 V_{2}\right)$ \\
13 & $24170 V_{0}+16490 V_{1}+35427 V_{2}$ & -
\end{tabular}

The first few terms of the binomial transform numbers of the Narayana, Narayana-Lucas, Narayana-Perrin sequences with positive subscript and negative subscript are given in the following Table 2 . 
Table 2: A few binomial transform (terms).

\begin{tabular}{cccccccccccccc}
\hline$n$ & 0 & 1 & 2 & 3 & 4 & 5 & 6 & 7 & 8 & 9 & 10 & 11 & 12 \\
\hline$\widehat{N}_{n}$ & 0 & 1 & 3 & 7 & 16 & 38 & 93 & 230 & 569 & 1405 & 3465 & 8542 & 21058 \\
$\widehat{N}_{-n}$ & & $-\frac{1}{3}$ & $-\frac{2}{9}$ & $\frac{2}{27}$ & $\frac{25}{81}$ & $\frac{83}{243}$ & $\frac{133}{729}$ & $-\frac{106}{2187}$ & $-\frac{1379}{6561}$ & $-\frac{4426}{19683}$ & $-\frac{6536}{59049}$ & $\frac{8021}{177147}$ & $\frac{78703}{531441}$ \\
$\widehat{U}_{n}$ & 3 & 4 & 6 & 13 & 34 & 89 & 225 & 557 & 1370 & 3370 & 8301 & 20464 & 50461 \\
$\widehat{U}_{-n}$ & & $\frac{5}{3}$ & $\frac{1}{9}$ & $-\frac{28}{27}$ & $-\frac{107}{81}$ & $-\frac{190}{243}$ & $\frac{82}{729}$ & $\frac{1727}{2187}$ & $\frac{5941}{6561}$ & $\frac{9719}{19683}$ & $-\frac{7154}{59049}$ & $-\frac{98929}{177147}$ & $-\frac{321326}{531441}$ \\
$\widehat{H}_{n}$ & 3 & 3 & 5 & 14 & 40 & 105 & 262 & 643 & 1577 & 3879 & 9560 & 23576 & 58141 \\
$\widehat{H}_{-n}$ & & $\frac{8}{3}$ & $\frac{13}{9}$ & $-\frac{4}{27}$ & $-\frac{104}{81}$ & $-\frac{355}{243}$ & $-\frac{563}{729}$ & $\frac{509}{2187}$ & $\frac{6106}{6561}$ & $\frac{19355}{19683}$ & $\frac{28084}{59049}$ & $-\frac{36886}{177147}$ & $-\frac{347243}{531441}$ \\
\hline
\end{tabular}

(1.3) can be used to obtain Binet's formula of the binomial transform of generalized Narayana numbers. Binet's formula of the binomial transform of generalized Narayana numbers can be given as

$$
b_{n}=\frac{c_{1} \theta_{1}^{n}}{\left(\theta_{1}-\theta_{2}\right)\left(\theta_{1}-\theta_{3}\right)}+\frac{c_{2} \theta_{2}^{n}}{\left(\theta_{2}-\theta_{1}\right)\left(\theta_{2}-\theta_{3}\right)}+\frac{c_{3} \theta_{3}^{n}}{\left(\theta_{3}-\theta_{1}\right)\left(\theta_{3}-\theta_{2}\right)}
$$

where

$$
\begin{aligned}
& c_{1}=b_{2}-\left(\theta_{2}+\theta_{3}\right) b_{1}+\theta_{2} \theta_{3} b_{0}=\left(V_{0}+2 V_{1}+V_{2}\right)-\left(\theta_{2}+\theta_{3}\right)\left(V_{0}+V_{1}\right)+\theta_{2} \theta_{3} V_{0}, \\
& c_{2}=b_{2}-\left(\theta_{1}+\theta_{3}\right) b_{1}+\theta_{1} \theta_{3} b_{0}=\left(V_{0}+2 V_{1}+V_{2}\right)-\left(\theta_{1}+\theta_{3}\right)\left(V_{0}+V_{1}\right)+\theta_{1} \theta_{3} V_{0}, \\
& c_{3}=b_{2}-\left(\theta_{1}+\theta_{2}\right) b_{1}+\theta_{1} \theta_{2} b_{0}=\left(V_{0}+2 V_{1}+V_{2}\right)-\left(\theta_{1}+\theta_{2}\right)\left(V_{0}+V_{1}\right)+\theta_{1} \theta_{2} V_{0} .
\end{aligned}
$$

Here, $\theta_{1}, \theta_{2}$ and $\theta_{3}$ are the roots of the cubic equation $x^{3}-4 x^{2}+5 x-3=0$. Moreover,

$$
\begin{aligned}
& \theta_{1}=\frac{4}{3}+\frac{1}{6} \sqrt[3]{4(29+3 \sqrt{93})}+\frac{1}{6} \sqrt[3]{4(29-3 \sqrt{93})} \\
& \theta_{2}=\frac{4}{3}+\frac{\omega}{6} \sqrt[3]{4(29+3 \sqrt{93})}+\frac{\omega^{2}}{6} \sqrt[3]{4(29-3 \sqrt{93})} \\
& \theta_{3}=\frac{4}{3}+\frac{\omega^{2}}{6} \sqrt[3]{4(29+3 \sqrt{93})}+\frac{\omega}{6} \sqrt[3]{4(29-3 \sqrt{93})}
\end{aligned}
$$

where

$$
\omega=\frac{-1+i \sqrt{3}}{2}=\exp (2 \pi i / 3)
$$


Note that

$$
\begin{aligned}
\theta_{1}+\theta_{2}+\theta_{3} & =4, \\
\theta_{1} \theta_{2}+\theta_{1} \theta_{3}+\theta_{2} \theta_{3} & =5, \\
\theta_{1} \theta_{2} \theta_{3} & =3 .
\end{aligned}
$$

For all integers $n$, (Binet's formulas of) binomial transforms of Narayana , Narayana-Lucas, Narayana-Perrin numbers (using initial conditions in (2.2p) can be expressed using Binet's formulas as

$$
\begin{aligned}
\widehat{N}_{n} & =\frac{\left(-1+\theta_{1}\right) \theta_{1}^{n}}{\left(\theta_{1}-\theta_{2}\right)\left(\theta_{1}-\theta_{3}\right)}+\frac{\left(-1+\theta_{2}\right) \theta_{2}^{n}}{\left(\theta_{2}-\theta_{1}\right)\left(\theta_{2}-\theta_{3}\right)}+\frac{\left(-1+\theta_{3}\right) \theta_{3}^{n}}{\left(\theta_{3}-\theta_{1}\right)\left(\theta_{3}-\theta_{2}\right)}, \\
\widehat{U}_{n} & =\theta_{1}^{n}+\theta_{2}^{n}+\theta_{3}^{n}, \\
\widehat{H}_{n} & =\frac{\left(3 \theta_{1}^{2}-7 \theta_{1}+9\right) \theta_{1}^{n-1}}{\left(\theta_{1}-\theta_{2}\right)\left(\theta_{1}-\theta_{3}\right)}+\frac{\left(3 \theta_{2}^{2}-7 \theta_{2}+9\right) \theta_{2}^{n-1}}{\left(\theta_{2}-\theta_{1}\right)\left(\theta_{2}-\theta_{3}\right)}+\frac{\left(3 \theta_{3}^{2}-7 \theta_{3}+9\right) \theta_{3}^{n-1}}{\left(\theta_{3}-\theta_{1}\right)\left(\theta_{3}-\theta_{2}\right)},
\end{aligned}
$$

respectively.

\section{Generating Functions and Obtaining Binet Formula of Binomial Transform From Generating Function}

The generating function of the binomial transform of the generalized Narayana sequence $V_{n}$ is a power series centered at the origin whose coefficients are the binomial transform of the generalized Narayana sequence.

Next, we give the ordinary generating function $f_{b_{n}}(x)=\sum_{n=0}^{\infty} b_{n} x^{n}$ of the sequence $b_{n}$.

Lemma 3.1. Suppose that $f_{b_{n}}(x)=\sum_{n=0}^{\infty} b_{n} x^{n}$ is the ordinary generating function of the binomial transform of the generalized Narayana sequence $\left\{V_{n}\right\}_{n \geq 0}$. Then, $f_{b_{n}}(x)$ is given by

$$
f_{b_{n}}(x)=\frac{V_{0}+\left(V_{1}-3 V_{0}\right) x+\left(2 V_{0}-2 V_{1}+V_{2}\right) x^{2}}{1-4 x+5 x^{2}-3 x^{3}} .
$$


Proof. Using Lemma 1.1, we obtain

$$
\begin{aligned}
f_{b_{n}}(x) & =\frac{b_{0}+\left(b_{1}-r_{1} b_{0}\right) x+\left(b_{2}-r_{1} b_{1}-s_{1} b_{0}\right) x^{2}}{1-r_{1} x-s_{1} x^{2}-t_{1} x^{3}} \\
& =\frac{V_{0}+\left(\left(V_{0}+V_{1}\right)-4 V_{0}\right) x+\left(\left(V_{0}+2 V_{1}+V_{2}\right)-4\left(V_{0}+V_{1}\right)-(-5) V_{0}\right) x^{2}}{1-4 x-(-5) x^{2}-3 x^{3}} \\
& =\frac{V_{0}+\left(V_{1}-3 V_{0}\right) x+\left(2 V_{0}-2 V_{1}+V_{2}\right) x^{2}}{1-4 x+5 x^{2}-3 x^{3}}
\end{aligned}
$$

where

$$
\begin{aligned}
& b_{0}=V_{0}, \\
& b_{1}=V_{0}+V_{1}, \\
& b_{2}=V_{0}+2 V_{1}+V_{2} .
\end{aligned}
$$

Note that P. Barry shows in 1 that if $A(x)$ is the generating function of the sequence $\left\{a_{n}\right\}$, then

$$
S(x)=\frac{1}{1-x} A\left(\frac{x}{1-x}\right)
$$

is the generating function of the sequence $\left\{b_{n}\right\}$ with $b_{n}=\sum_{i=0}^{n}\left(\begin{array}{c}n \\ i\end{array}\right) a_{i}$. In our case, since

$$
A(x)=\frac{V_{0}+\left(V_{1}-V_{0}\right) x+\left(V_{2}-V_{1}\right) x^{2}}{1-x-x^{3}}, \quad \text { see } 1.10,
$$

we obtain

$$
\begin{aligned}
S(x) & =\frac{1}{1-x} \frac{V_{0}+\left(V_{1}-V_{0}\right)\left(\frac{x}{1-x}\right)+\left(V_{2}-V_{1}\right)\left(\frac{x}{1-x}\right)^{2}}{1-\left(\frac{x}{1-x}\right)-\left(\frac{x}{1-x}\right)^{3}} \\
& =\frac{V_{0}+\left(V_{1}-3 V_{0}\right) x+\left(2 V_{0}-2 V_{1}+V_{2}\right) x^{2}}{1-4 x+5 x^{2}-3 x^{3}} .
\end{aligned}
$$

The previous lemma gives the following results as particular examples. 
Corollary 3.2. Generating functions of the binomial transform of the Narayana, Narayana-Lucas, Narayana-Perrin numbers are

$$
\begin{aligned}
\sum_{n=0}^{\infty} \widehat{N}_{n} x^{n} & =\frac{x-x^{2}}{1-4 x+5 x^{2}-3 x^{3}}, \\
\sum_{n=0}^{\infty} \widehat{U}_{n} x^{n} & =\frac{3-8 x+5 x^{2}}{1-4 x+5 x^{2}-3 x^{3}}, \\
\sum_{n=0}^{\infty} \widehat{H}_{n} x^{n} & =\frac{3-9 x+8 x^{2}}{1-4 x+5 x^{2}-3 x^{3}},
\end{aligned}
$$

respectively.

We next find Binet's formula of the Binomial transform of the generalized Narayana numbers $\left\{V_{n}\right\}$ by the use of generating function for $b_{n}$.

Theorem 3.3. (Binet's formula of the Binomial transform of the generalized Narayana numbers)

$$
b_{n}=\frac{d_{1} \theta_{1}^{n}}{\left(\theta_{1}-\theta_{2}\right)\left(\theta_{1}-\theta_{3}\right)}+\frac{d_{2} \theta_{2}^{n}}{\left(\theta_{2}-\theta_{1}\right)\left(\theta_{2}-\theta_{3}\right)}+\frac{d_{3} \theta_{3}^{n}}{\left(\theta_{3}-\theta_{1}\right)\left(\theta_{3}-\theta_{2}\right)}
$$

where

$$
\begin{aligned}
& d_{1}=V_{0} \theta_{1}^{2}+\left(V_{1}-3 V_{0}\right) \theta_{1}+\left(2 V_{0}-2 V_{1}+V_{2}\right), \\
& d_{2}=V_{0} \theta_{2}^{2}+\left(V_{1}-3 V_{0}\right) \theta_{2}+\left(2 V_{0}-2 V_{1}+V_{2}\right), \\
& d_{3}=V_{0} \theta_{3}^{2}+\left(V_{1}-3 V_{0}\right) \theta_{3}+\left(2 V_{0}-2 V_{1}+V_{2}\right) .
\end{aligned}
$$

Proof. By using Lemma 3.1, the proof follows from Theorem 1.2 .

Note that from (2.2) and (3.2), we have

$$
\begin{aligned}
& b_{2}-\left(\theta_{2}+\theta_{3}\right) b_{1}+\theta_{2} \theta_{3} b_{0}=V_{0} \theta_{1}^{2}+\left(V_{1}-3 V_{0}\right) \theta_{1}+\left(2 V_{0}-2 V_{1}+V_{2}\right), \\
& b_{2}-\left(\theta_{1}+\theta_{3}\right) b_{1}+\theta_{1} \theta_{3} b_{0}=V_{0} \theta_{2}^{2}+\left(V_{1}-3 V_{0}\right) \theta_{2}+\left(2 V_{0}-2 V_{1}+V_{2}\right), \\
& b_{2}-\left(\theta_{1}+\theta_{2}\right) b_{1}+\theta_{1} \theta_{2} b_{0}=V_{0} \theta_{3}^{2}+\left(V_{1}-3 V_{0}\right) \theta_{3}+\left(2 V_{0}-2 V_{1}+V_{2}\right),
\end{aligned}
$$


or

$$
\begin{aligned}
\left(V_{0}+2 V_{1}+V_{2}\right)-\left(\theta_{2}+\theta_{3}\right)\left(V_{0}+V_{1}\right)+\theta_{2} \theta_{3} V_{0}= & V_{0} \theta_{1}^{2}+\left(V_{1}-3 V_{0}\right) \theta_{1} \\
& +\left(2 V_{0}-2 V_{1}+V_{2}\right), \\
\left(V_{0}+2 V_{1}+V_{2}\right)-\left(\theta_{1}+\theta_{3}\right)\left(V_{0}+V_{1}\right)+\theta_{1} \theta_{3} V_{0}= & V_{0} \theta_{2}^{2}+\left(V_{1}-3 V_{0}\right) \theta_{2} \\
& +\left(2 V_{0}-2 V_{1}+V_{2}\right), \\
\left(V_{0}+2 V_{1}+V_{2}\right)-\left(\theta_{1}+\theta_{2}\right)\left(V_{0}+V_{1}\right)+\theta_{1} \theta_{2} V_{0}= & V_{0} \theta_{3}^{2}+\left(V_{1}-3 V_{0}\right) \theta_{3} \\
& +\left(2 V_{0}-2 V_{1}+V_{2}\right) .
\end{aligned}
$$

Note that we can also write

$$
\begin{aligned}
\left(b_{0}+2 b_{1}+b_{2}\right)-\left(\theta_{2}+\theta_{3}\right)\left(b_{0}+b_{1}\right)+\theta_{2} \theta_{3} b_{0}= & b_{0} \theta_{1}^{2}+\left(b_{1}-3 b_{0}\right) \theta_{1} \\
& +\left(2 b_{0}-2 b_{1}+b_{2}\right), \\
\left(b_{0}+2 b_{1}+b_{2}\right)-\left(\theta_{1}+\theta_{3}\right)\left(b_{0}+b_{1}\right)+\theta_{1} \theta_{3} b_{0}= & b_{0} \theta_{2}^{2}+\left(b_{1}-3 b_{0}\right) \theta_{2} \\
& +\left(2 b_{0}-2 b_{1}+b_{2}\right), \\
\left(b_{0}+2 b_{1}+b_{2}\right)-\left(\theta_{1}+\theta_{2}\right)\left(b_{0}+b_{1}\right)+\theta_{1} \theta_{2} b_{0}= & b_{0} \theta_{3}^{2}+\left(b_{1}-3 b_{0}\right) \theta_{3} \\
& +\left(2 b_{0}-2 b_{1}+b_{2}\right) .
\end{aligned}
$$

Next, using Theorem 3.3, we present the Binet's formulas of binomial transform of Narayana, Narayana-Lucas, Narayana-Perrin sequences.

Corollary 3.4. Binet's formulas of binomial transform of Narayana, Narayana-Lucas, Narayana-Perrin sequences are

$$
\begin{aligned}
\widehat{N}_{n} & =\frac{\left(-1+\theta_{1}\right) \theta_{1}^{n}}{\left(\theta_{1}-\theta_{2}\right)\left(\theta_{1}-\theta_{3}\right)}+\frac{\left(-1+\theta_{2}\right) \theta_{2}^{n}}{\left(\theta_{2}-\theta_{1}\right)\left(\theta_{2}-\theta_{3}\right)}+\frac{\left(-1+\theta_{3}\right) \theta_{3}^{n}}{\left(\theta_{3}-\theta_{1}\right)\left(\theta_{3}-\theta_{2}\right)}, \\
\widehat{U}_{n} & =\theta_{1}^{n}+\theta_{2}^{n}+\theta_{3}^{n} \\
\widehat{H}_{n} & =\frac{\left(3 \theta_{1}^{2}-7 \theta_{1}+9\right) \theta_{1}^{n-1}}{\left(\theta_{1}-\theta_{2}\right)\left(\theta_{1}-\theta_{3}\right)}+\frac{\left(3 \theta_{2}^{2}-7 \theta_{2}+9\right) \theta_{2}^{n-1}}{\left(\theta_{2}-\theta_{1}\right)\left(\theta_{2}-\theta_{3}\right)}+\frac{\left(3 \theta_{3}^{2}-7 \theta_{3}+9\right) \theta_{3}^{n-1}}{\left(\theta_{3}-\theta_{1}\right)\left(\theta_{3}-\theta_{2}\right)},
\end{aligned}
$$

respectively. 


\section{Simson Formulas}

There is a well-known Simson Identity (formula) for Fibonacci sequence $\left\{F_{n}\right\}$, namely,

$$
F_{n+1} F_{n-1}-F_{n}^{2}=(-1)^{n}
$$

which was derived first by R. Simson in 1753 and it is now called as Cassini Identity (formula) as well. This can be written in the form

$$
\left|\begin{array}{cc}
F_{n+1} & F_{n} \\
F_{n} & F_{n-1}
\end{array}\right|=(-1)^{n} .
$$

The following theorem gives generalization of this result to the generalized Narayana sequence $\left\{W_{n}\right\}$.

Theorem 4.1 (Simson Formula of Generalized Tribonacci Numbers). For all integers $n$, we have

$$
\left|\begin{array}{ccc}
W_{n+2} & W_{n+1} & W_{n} \\
W_{n+1} & W_{n} & W_{n-1} \\
W_{n} & W_{n-1} & W_{n-2}
\end{array}\right|=t^{n}\left|\begin{array}{ccc}
W_{2} & W_{1} & W_{0} \\
W_{1} & W_{0} & W_{-1} \\
W_{0} & W_{-1} & W_{-2}
\end{array}\right| .
$$

Proof. 4.1 is given in Soykan 22.

Taking $\left\{W_{n}\right\}=\left\{b_{n}\right\}$ in the above theorem and considering $b_{n+3}=4 b_{n+2}-$ $5 b_{n+1}+3 b_{n}, r=4, s=-5, t=3$, we have the following proposition.

Proposition 4.2. For all integers n, Simson formula of binomial transforms of generalized Narayana numbers is given as

$$
\left|\begin{array}{ccc}
b_{n+2} & b_{n+1} & b_{n} \\
b_{n+1} & b_{n} & b_{n-1} \\
b_{n} & b_{n-1} & b_{n-2}
\end{array}\right|=3^{n}\left|\begin{array}{ccc}
b_{2} & b_{1} & b_{0} \\
b_{1} & b_{0} & b_{-1} \\
b_{0} & b_{-1} & b_{-2}
\end{array}\right| .
$$

The previous proposition gives the following results as particular examples. 
Corollary 4.3. For all integers n, Simson formula of binomial transforms of the Narayana, Narayana-Lucas, Narayana-Perrin numbers are given as

$$
\begin{aligned}
& \left|\begin{array}{ccc}
\widehat{N}_{n+2} & \widehat{N}_{n+1} & \widehat{N}_{n} \\
\widehat{N}_{n+1} & \widehat{N}_{n} & \widehat{N}_{n-1} \\
\widehat{N}_{n} & \widehat{N}_{n-1} & \widehat{N}_{n-2}
\end{array}\right|=-3^{n-2}, \\
& \left|\begin{array}{ccc}
\widehat{U}_{n+2} & \widehat{U}_{n+1} & \widehat{U}_{n} \\
\widehat{U}_{n+1} & \widehat{U}_{n} & \widehat{U}_{n-1} \\
\widehat{U}_{n} & \widehat{U}_{n-1} & \widehat{U}_{n-2}
\end{array}\right|=-31 \times 3^{n-2}, \\
& \left|\begin{array}{ccc}
\widehat{H}_{n+2} & \widehat{H}_{n+1} & \widehat{H}_{n} \\
\widehat{H}_{n+1} & \widehat{H}_{n} & \widehat{H}_{n-1} \\
\widehat{H}_{n} & \widehat{H}_{n-1} & \widehat{H}_{n-2}
\end{array}\right|=-53 \times 3^{n-2},
\end{aligned}
$$

respectively.

\section{Some Identities}

In this section, we obtain some identities of binomial transforms of Narayana, Narayana-Lucas, Narayana-Perrin numbers. First, we can give a few basic relations between $\left\{\widehat{N}_{n}\right\}$ and $\left\{\widehat{U}_{n}\right\}$.

Lemma 5.1. The following equalities are true:

$$
\begin{aligned}
279 \widehat{N}_{n} & =-13 \widehat{U}_{n+4}+82 \widehat{U}_{n+3}-104 \widehat{U}_{n+2} \\
93 \widehat{N}_{n} & =10 \widehat{U}_{n+3}-13 \widehat{U}_{n+2}-13 \widehat{U}_{n+1} \\
31 \widehat{N}_{n} & =9 \widehat{U}_{n+2}-21 \widehat{U}_{n+1}+10 \widehat{U}_{n} \\
31 \widehat{N}_{n} & =15 \widehat{U}_{n+1}-35 \widehat{U}_{n}+27 \widehat{U}_{n-1} \\
31 \widehat{N}_{n} & =25 \widehat{U}_{n}-48 \widehat{U}_{n-1}+45 \widehat{U}_{n-2}
\end{aligned}
$$


and

$$
\begin{aligned}
9 \widehat{U}_{n} & =-16 \widehat{N}_{n+4}+49 \widehat{N}_{n+3}-20 \widehat{N}_{n+2} \\
3 \widehat{U}_{n} & =-5 \widehat{N}_{n+3}+20 \widehat{N}_{n+2}-16 \widehat{N}_{n+1} \\
\widehat{U}_{n} & =3 \widehat{N}_{n+1}-5 \widehat{N}_{n} \\
\widehat{U}_{n} & =7 \widehat{N}_{n}-15 \widehat{N}_{n-1}+9 \widehat{N}_{n-2} .
\end{aligned}
$$

Proof. Note that all the identities hold for all integers $n$. We prove (5.1). To show (5.1), writing

$$
\widehat{N}_{n}=a \times \widehat{U}_{n+4}+b \times \widehat{U}_{n+3}+c \times \widehat{U}_{n+2}
$$

and solving the system of equations

$$
\begin{aligned}
& \widehat{N}_{0}=a \times \widehat{U}_{4}+b \times \widehat{U}_{3}+c \times \widehat{U}_{2} \\
& \widehat{N}_{1}=a \times \widehat{U}_{5}+b \times \widehat{U}_{4}+c \times \widehat{U}_{3} \\
& \widehat{N}_{2}=a \times \widehat{U}_{6}+b \times \widehat{U}_{5}+c \times \widehat{U}_{4}
\end{aligned}
$$

we find that $a=-\frac{13}{279}, b=\frac{82}{279}, c=-\frac{104}{279}$. The other equalities can be proved similarly.

Note that all the identities in the above Lemma can be proved by induction as well.

Next, we present a few basic relations between $\left\{\widehat{N}_{n}\right\}$ and $\left\{\widehat{H}_{n}\right\}$.

Lemma 5.2. The following equalities are true:

$$
\begin{aligned}
477 \widehat{N}_{n} & =-47 \widehat{H}_{n+4}+185 \widehat{H}_{n+3}-142 \widehat{H}_{n+2}, \\
159 \widehat{N}_{n} & =-\widehat{H}_{n+3}+31 \widehat{H}_{n+2}-47 \widehat{H}_{n+1}, \\
53 \widehat{N}_{n} & =9 \widehat{H}_{n+2}-14 \widehat{H}_{n+1}-\widehat{H}_{n} \\
53 \widehat{N}_{n} & =22 \widehat{H}_{n+1}-46 \widehat{H}_{n}+27 \widehat{H}_{n-1}, \\
53 \widehat{N}_{n} & =42 \widehat{H}_{n}-83 \widehat{H}_{n-1}+66 \widehat{H}_{n-2}
\end{aligned}
$$


and

$$
\begin{aligned}
9 \widehat{H}_{n} & =-19 \widehat{N}_{n+4}+70 \widehat{N}_{n+3}-53 \widehat{N}_{n+2} \\
3 \widehat{H}_{n} & =-2 \widehat{N}_{n+3}+14 \widehat{N}_{n+2}-19 \widehat{N}_{n+1} \\
\widehat{H}_{n} & =2 \widehat{N}_{n+2}-3 \widehat{N}_{n+1}-2 \widehat{N}_{n} \\
\widehat{H}_{n} & =5 \widehat{N}_{n+1}-12 \widehat{N}_{n}+6 \widehat{N}_{n-1} \\
\widehat{H}_{n} & =8 \widehat{N}_{n}-19 \widehat{N}_{n-1}+15 \widehat{N}_{n-2}
\end{aligned}
$$

Now, we give a few basic relations between $\left\{\widehat{U}_{n}\right\}$ and $\left\{\widehat{H}_{n}\right\}$.

Lemma 5.3. The following equalities are true:

$$
\begin{aligned}
477 \widehat{U}_{n} & =226 \widehat{H}_{n+4}-646 \widehat{H}_{n+3}+287 \widehat{H}_{n+2} \\
159 \widehat{U}_{n} & =86 \widehat{H}_{n+3}-281 \widehat{H}_{n+2}+226 \widehat{H}_{n+1} \\
53 \widehat{U}_{n} & =21 \widehat{H}_{n+2}-68 \widehat{H}_{n+1}+86 \widehat{H}_{n} \\
53 \widehat{U}_{n} & =16 \widehat{H}_{n+1}-19 \widehat{H}_{n}+63 \widehat{H}_{n-1} \\
53 \widehat{U}_{n} & =45 \widehat{H}_{n}-17 \widehat{H}_{n-1}+48 \widehat{H}_{n-2}
\end{aligned}
$$

and

$$
\begin{aligned}
279 \widehat{H}_{n} & =98 \widehat{U}_{n+4}-425 \widehat{U}_{n+3}+505 \widehat{U}_{n+2}, \\
93 \widehat{H}_{n} & =-11 \widehat{U}_{n+3}+5 \widehat{U}_{n+2}+98 \widehat{U}_{n+1}, \\
31 \widehat{H}_{n} & =-13 \widehat{U}_{n+2}+51 \widehat{U}_{n+1}-11 \widehat{U}_{n}, \\
31 \widehat{H}_{n} & =-\widehat{U}_{n+1}+54 \widehat{U}_{n}-39 \widehat{U}_{n-1}, \\
31 \widehat{H}_{n} & =50 \widehat{U}_{n}-34 \widehat{U}_{n-1}-3 \widehat{U}_{n-2} .
\end{aligned}
$$

\section{Sum Formulas}

\subsection{Sums of Terms with Positive Subscripts}

The following proposition presents some formulas of binomial transform of generalized Narayana numbers with positive subscripts. 
Proposition 6.1. For $n \geq 0$, we have the following formulas:

(a) $\sum_{k=0}^{n} b_{k}=b_{n+3}-3 b_{n+2}+2 b_{n+1}-b_{2}+3 b_{1}-2 b_{0}$.

(b) $\sum_{k=0}^{n} b_{2 k}=\frac{1}{13}\left(6 b_{2 n+2}-17 b_{2 n+1}+21 b_{2 n}-6 b_{2}+17 b_{1}-8 b_{0}\right)$.

(c) $\sum_{k=0}^{n} b_{2 k+1}=\frac{1}{13}\left(7 b_{2 n+2}-9 b_{2 n+1}+18 b_{2 n}-7 b_{2}+22 b_{1}-18 b_{0}\right)$.

Proof. Take $r=4, s=-5, t=3$ in Theorem 2.1 in 24 (or take $x=1, r=4, s=$ $-5, t=3$ in Theorem 2.1 in 25 ).

From the last proposition, we have the following corollary which gives sum formulas of binomial transform of Narayana numbers (take $b_{n}=\widehat{N}_{n}$ with $\widehat{N}_{0}=$ $\left.0, \widehat{N}_{1}=1, \widehat{N}_{2}=3\right)$.

Corollary 6.2. For $n \geq 0$ we have the following formulas:

(a) $\sum_{k=0}^{n} \widehat{N}_{k}=\widehat{N}_{n+3}-3 \widehat{N}_{n+2}+2 \widehat{N}_{n+1}$.

(b) $\sum_{k=0}^{n} \widehat{N}_{2 k}=\frac{1}{13}\left(6 \widehat{N}_{2 n+2}-17 \widehat{N}_{2 n+1}+21 \widehat{N}_{2 n}-1\right)$.

(c) $\sum_{k=0}^{n} \widehat{N}_{2 k+1}=\frac{1}{13}\left(7 \widehat{N}_{2 n+2}-9 \widehat{N}_{2 n+1}+18 \widehat{N}_{2 n}+1\right)$.

Taking $b_{n}=\widehat{U}_{n}$ with $\widehat{U}_{0}=3, \widehat{U}_{1}=4, \widehat{U}_{2}=6$ in the last proposition, we have the following corollary which presents sum formulas of binomial transform of Narayana-Lucas numbers.

Corollary 6.3. For $n \geq 0$ we have the following formulas:

(a) $\sum_{k=0}^{n} \widehat{U}_{k}=\widehat{U}_{n+3}-3 \widehat{U}_{n+2}+2 \widehat{U}_{n+1}$.

(b) $\sum_{k=0}^{n} \widehat{U}_{2 k}=\frac{1}{13}\left(6 \widehat{U}_{2 n+2}-17 \widehat{U}_{2 n+1}+21 \widehat{U}_{2 n}+8\right)$.

(c) $\sum_{k=0}^{n} \widehat{U}_{2 k+1}=\frac{1}{13}\left(7 \widehat{U}_{2 n+2}-9 \widehat{U}_{2 n+1}+18 \widehat{U}_{2 n}-8\right)$.

From the last proposition, we have the following corollary which gives sum formulas of binomial transform of Narayana-Perrin numbers (take $b_{n}=\widehat{H}_{n}$ with $\widehat{H}_{0}=3, \widehat{H}_{1}=3, \widehat{H}_{2}=5$ ). 
Corollary 6.4. For $n \geq 0$ we have the following formulas:
(a) $\sum_{k=0}^{n} \widehat{H}_{k}=\widehat{H}_{n+3}-3 \widehat{H}_{n+2}+2 \widehat{H}_{n+1}-2$.
(b) $\sum_{k=0}^{n} \widehat{H}_{2 k}=\frac{1}{13}\left(6 \widehat{H}_{2 n+2}-17 \widehat{H}_{2 n+1}+21 \widehat{H}_{2 n}-3\right)$.
(c) $\sum_{k=0}^{n} \widehat{H}_{2 k+1}=\frac{1}{13}\left(7 \widehat{H}_{2 n+2}-9 \widehat{H}_{2 n+1}+18 \widehat{H}_{2 n}-23\right)$.

\subsection{Sums of Terms with Negative Subscripts}

The following proposition presents some formulas of binomial transform of generalized Narayana numbers with negative subscripts.

Proposition 6.5. For $n \geq 1$ we have the following formulas:

(a) $\sum_{k=1}^{n} b_{-k}=-2 b_{-n-1}+2 b_{-n-2}-3 b_{-n-3}+b_{2}-3 b_{1}+2 b_{0}$.

(b) $\sum_{k=1}^{n} b_{-2 k}=\frac{1}{13}\left(-7 b_{-2 n+1}+22 b_{-2 n}-18 b_{-2 n-1}+6 b_{2}-17 b_{1}+8 b_{0}\right)$.

(c) $\sum_{k=1}^{n} b_{-2 k+1}=\frac{1}{13}\left(-6 b_{-2 n+1}+17 b_{-2 n}-21 b_{-2 n-1}+7 b_{2}-22 b_{1}+18 b_{0}\right)$.

Proof. Take $r=4, s=-5, t=3$ in Theorem 3.1 in 24 or (or take $x=1, r=$ $4, s=-5, t=3$ in Theorem 3.1 in [25]).

From the last proposition, we have the following corollary which gives sum formulas of binomial transform of Narayana numbers (take $b_{n}=\widehat{N}_{n}$ with $\widehat{N}_{0}=$ $\left.0, \widehat{N}_{1}=1, \widehat{N}_{2}=3\right)$.

Corollary 6.6. For $n \geq 1$, binomial transform of Narayana numbers have the following properties.

(a) $\sum_{k=1}^{n} \widehat{N}_{-k}=-2 \widehat{N}_{-n-1}+2 \widehat{N}_{-n-2}-3 \widehat{N}_{-n-3}$.

(b) $\sum_{k=1}^{n} \widehat{N}_{-2 k}=\frac{1}{13}\left(-7 \widehat{N}_{-2 n+1}+22 \widehat{N}_{-2 n}-18 \widehat{N}_{-2 n-1}+1\right)$.

(c) $\sum_{k=1}^{n} \widehat{N}_{-2 k+1}=\frac{1}{13}\left(-6 \widehat{N}_{-2 n+1}+17 \widehat{N}_{-2 n}-21 \widehat{N}_{-2 n-1}-1\right)$.

Taking $b_{n}=\widehat{U}_{n}$ with $\widehat{U}_{0}=3, \widehat{U}_{1}=4, \widehat{U}_{2}=6$ in the last proposition, we have the following corollary which presents sum formulas of binomial transform of Narayana-Lucas numbers. 
Corollary 6.7. For $n \geq 1$, binomial transform of Narayana-Lucas numbers have the following properties.

(a) $\sum_{k=1}^{n} \widehat{U}_{-k}=-2 \widehat{U}_{-n-1}+2 \widehat{U}_{-n-2}-3 \widehat{U}_{-n-3}$.

(b) $\sum_{k=1}^{n} \widehat{U}_{-2 k}=\frac{1}{13}\left(-7 \widehat{U}_{-2 n+1}+22 \widehat{U}_{-2 n}-18 \widehat{U}_{-2 n-1}-8\right)$.

(c) $\sum_{k=1}^{n} \widehat{U}_{-2 k+1}=\frac{1}{13}\left(-6 \widehat{U}_{-2 n+1}+17 \widehat{U}_{-2 n}-21 \widehat{U}_{-2 n-1}+8\right)$.

From the last proposition, we have the following corollary which gives sum formulas of binomial transform of Narayana-Perrin numbers (take $b_{n}=\widehat{H}_{n}$ with $\widehat{H}_{0}=3, \widehat{H}_{1}=3, \widehat{H}_{2}=5$ ).

Corollary 6.8. For $n \geq 1$, binomial transform of Narayana-Perrin numbers have the following properties.
(a) $\sum_{k=1}^{n} \widehat{H}_{-k}=-2 \widehat{H}_{-n-1}+2 \widehat{H}_{-n-2}-3 \widehat{H}_{-n-3}+2$.
(b) $\sum_{k=1}^{n} \widehat{H}_{-2 k}=\frac{1}{13}\left(-7 \widehat{H}_{-2 n+1}+22 \widehat{H}_{-2 n}-18 \widehat{H}_{-2 n-1}+3\right)$.
(c) $\sum_{k=1}^{n} \widehat{H}_{-2 k+1}=\frac{1}{13}\left(-6 \widehat{H}_{-2 n+1}+17 \widehat{H}_{-2 n}-21 \widehat{H}_{-2 n-1}+23\right)$.

\subsection{Sums of Squares of Terms with Positive Subscripts}

The following proposition presents some formulas of binomial transform of generalized Narayana numbers with positive subscripts.

Proposition 6.9. For $n \geq 0$, we have the following formulas:

(a) $\sum_{k=0}^{n} b_{k}^{2}=\frac{1}{13}\left(15 b_{n+3}^{2}+167 b_{n+2}^{2}+122 b_{n+1}^{2}-98 b_{n+3} b_{n+2}+66 b_{n+3} b_{n+1}-\right.$ $\left.252 b_{n+2} b_{n+1}-15 b_{2}^{2}-167 b_{1}^{2}-122 b_{0}^{2}+98 b_{2} b_{1}-66 b_{2} b_{0}+252 b_{1} b_{0}\right)$.

(b) $\sum_{k=0}^{n} b_{k+1} b_{k}=\frac{1}{13}\left(11 b_{n+3}^{2}+119 b_{n+2}^{2}+99 b_{n+1}^{2}-71 b_{n+3} b_{n+2}-190 b_{n+2} b_{n+1}+\right.$ $\left.51 b_{n+3} b_{n+1}-11 b_{2}^{2}-119 b_{1}^{2}-99 b_{0}^{2}+71 b_{2} b_{1}-51 b_{2} b_{0}+190 b_{1} b_{0}\right)$.

(c) $\sum_{k=0}^{n} b_{k+2} b_{k}=\frac{1}{13}\left(2 b_{n+3}^{2}-2 b_{n+2}^{2}+18 b_{n+1}^{2}-7 b_{n+3} b_{n+2}+14 b_{n+3} b_{n+1}-\right.$ $\left.18 b_{n+2} b_{n+1}-2 b_{2}^{2}+2 b_{1}^{2}-18 b_{0}^{2}+7 b_{2} b_{1}-14 b_{2} b_{0}+18 b_{1} b_{0}\right)$. 
Proof. Take $x=1, r=4, s=-5, t=3$ in Theorem 4.1 in 27, see also 26.

From the last proposition, we have the following Corollary which gives sum formulas of binomial transform of Narayana numbers (take $b_{n}=\widehat{N}_{n}$ with $\widehat{N}_{0}=$ $\left.0, \widehat{N}_{1}=1, \widehat{N}_{2}=3\right)$.

Corollary 6.10. For $n \geq 0$, binomial transform of Narayana numbers have the following properties:

(a) $\sum_{k=0}^{n} \widehat{N}_{k}^{2}=\frac{1}{13}\left(15 \widehat{N}_{n+3}^{2}+167 \widehat{N}_{n+2}^{2}+122 \widehat{N}_{n+1}^{2}-98 \widehat{N}_{n+3} \widehat{N}_{n+2}+66 \widehat{N}_{n+3} \widehat{N}_{n+1}-\right.$ $\left.252 \widehat{N}_{n+2} \widehat{N}_{n+1}-8\right)$.

(b) $\sum_{k=0}^{n} \widehat{N}_{k+1} \widehat{N}_{k}=\frac{1}{13}\left(11 \widehat{N}_{n+3}^{2}+119 \widehat{N}_{n+2}^{2}+99 \widehat{N}_{n+1}^{2}-71 \widehat{N}_{n+3} \widehat{N}_{n+2}-\right.$ $\left.190 \widehat{N}_{n+2} \widehat{N}_{n+1}+51 \widehat{N}_{n+3} \widehat{N}_{n+1}-5\right)$.

(c) $\sum_{k=0}^{n} \widehat{N}_{k+2} \widehat{N}_{k}=\frac{1}{13}\left(2 \widehat{N}_{n+3}^{2}-2 \widehat{N}_{n+2}^{2}+18 \widehat{N}_{n+1}^{2}-7 \widehat{N}_{n+3} \widehat{N}_{n+2}+14 \widehat{N}_{n+3} \widehat{N}_{n+1}-\right.$ $\left.18 \widehat{N}_{n+2} \widehat{N}_{n+1}+5\right)$.

Taking $b_{n}=\widehat{U}_{n}$ with $\widehat{U}_{0}=3, \widehat{U}_{1}=4, \widehat{U}_{2}=6$ in the last Proposition, we have the following Corollary which presents sum formulas of binomial transform of Narayana-Lucas numbers.

Corollary 6.11. For $n \geq 0$, binomial transform of Narayana-Lucas numbers have the following properties:

(a) $\sum_{k=0}^{n} \widehat{U}_{k}^{2}=\frac{1}{13}\left(15 \widehat{U}_{n+3}^{2}+167 \widehat{U}_{n+2}^{2}+122 \widehat{U}_{n+1}^{2}-98 \widehat{U}_{n+3} \widehat{U}_{n+2}+66 \widehat{U}_{n+3} \widehat{U}_{n+1}-\right.$ $\left.252 \widehat{U}_{n+2} \widehat{U}_{n+1}-122\right)$.

(b) $\sum_{k=0}^{n} \widehat{U}_{k+1} \widehat{U}_{k}=\frac{1}{13}\left(11 \widehat{U}_{n+3}^{2}+119 \widehat{U}_{n+2}^{2}+99 \widehat{U}_{n+1}^{2}-71 \widehat{U}_{n+3} \widehat{U}_{n+2}-\right.$ $\left.190 \widehat{U}_{n+2} \widehat{U}_{n+1}+51 \widehat{U}_{n+3} \widehat{U}_{n+1}-125\right)$.

(c) $\sum_{k=0}^{n} \widehat{U}_{k+2} \widehat{U}_{k}=\frac{1}{13}\left(2 \widehat{U}_{n+3}^{2}-2 \widehat{U}_{n+2}^{2}+18 \widehat{U}_{n+1}^{2}-7 \widehat{U}_{n+3} \widehat{U}_{n+2}+14 \widehat{U}_{n+3} \widehat{U}_{n+1}-\right.$ $\left.18 \widehat{U}_{n+2} \widehat{U}_{n+1}-70\right)$.

From the last proposition, we have the following corollary which gives sum formulas of binomial transform of Narayana-Perrin numbers (take $b_{n}=\widehat{H}_{n}$ with $\widehat{H}_{0}=3, \widehat{H}_{1}=3, \widehat{H}_{2}=5$ ). 
Corollary 6.12. For $n \geq 0$, binomial transform of Narayana-Perrin numbers have the following properties:

(a) $\sum_{k=0}^{n} \widehat{H}_{k}^{2}=\frac{1}{13}\left(15 \widehat{H}_{n+3}^{2}+167 \widehat{H}_{n+2}^{2}+122 \widehat{H}_{n+1}^{2}-98 \widehat{H}_{n+3} \widehat{H}_{n+2}+66 \widehat{H}_{n+3} \widehat{H}_{n+1}-\right.$ $\left.252 \widehat{H}_{n+2} \widehat{H}_{n+1}-228\right)$.

(b) $\sum_{k=0}^{n} \widehat{H}_{k+1} \widehat{H}_{k}=\frac{1}{13}\left(11 \widehat{H}_{n+3}^{2}+119 \widehat{H}_{n+2}^{2}+99 \widehat{H}_{n+1}^{2}-71 \widehat{H}_{n+3} \widehat{H}_{n+2}-\right.$ $\left.190 \widehat{H}_{n+2} \widehat{H}_{n+1}+51 \widehat{H}_{n+3} \widehat{H}_{n+1}-227\right)$.

(c) $\sum_{k=0}^{n} \widehat{H}_{k+2} \widehat{H}_{k}=\frac{1}{13}\left(2 \widehat{H}_{n+3}^{2}-2 \widehat{H}_{n+2}^{2}+18 \widehat{H}_{n+1}^{2}-7 \widehat{H}_{n+3} \widehat{H}_{n+2}+14 \widehat{H}_{n+3} \widehat{H}_{n+1}-\right.$ $\left.18 \widehat{H}_{n+2} \widehat{H}_{n+1}-137\right)$.

\section{Matrices related with Binomial Transform of Generalized Narayana numbers}

Matrix formulation of $W_{n}$ can be given as

$$
\left(\begin{array}{c}
W_{n+2} \\
W_{n+1} \\
W_{n}
\end{array}\right)=\left(\begin{array}{ccc}
r & s & t \\
1 & 0 & 0 \\
0 & 1 & 0
\end{array}\right)^{n}\left(\begin{array}{l}
W_{2} \\
W_{1} \\
W_{0}
\end{array}\right) .
$$

For matrix formulation (7.1), see [14. In fact, Kalman gave the formula in the following form

$$
\left(\begin{array}{c}
W_{n} \\
W_{n+1} \\
W_{n+2}
\end{array}\right)=\left(\begin{array}{lll}
0 & 1 & 0 \\
0 & 0 & 1 \\
r & s & t
\end{array}\right)^{n}\left(\begin{array}{c}
W_{0} \\
W_{1} \\
W_{2}
\end{array}\right) .
$$

We define the square matrix $A$ of order 3 as:

$$
A=\left(\begin{array}{ccc}
4 & -5 & 3 \\
1 & 0 & 0 \\
0 & 1 & 0
\end{array}\right)
$$


such that $\operatorname{det} A=3$. From 2.1 we have

$$
\left(\begin{array}{c}
b_{n+2} \\
b_{n+1} \\
b_{n}
\end{array}\right)=\left(\begin{array}{ccc}
4 & -5 & 3 \\
1 & 0 & 0 \\
0 & 1 & 0
\end{array}\right)\left(\begin{array}{c}
b_{n+1} \\
b_{n} \\
b_{n-1}
\end{array}\right)
$$

and from (7.1) (or using (7.2) and induction) we have

$$
\left(\begin{array}{c}
b_{n+2} \\
b_{n+1} \\
b_{n}
\end{array}\right)=\left(\begin{array}{ccc}
4 & -5 & 3 \\
1 & 0 & 0 \\
0 & 1 & 0
\end{array}\right)^{n}\left(\begin{array}{l}
b_{2} \\
b_{1} \\
b_{0}
\end{array}\right) .
$$

If we take $b_{n}=\widehat{N}_{n}$ in 7.2 we have

$$
\left(\begin{array}{c}
\widehat{N}_{n+2} \\
\widehat{N}_{n+1} \\
\widehat{N}_{n}
\end{array}\right)=\left(\begin{array}{ccc}
4 & -5 & 3 \\
1 & 0 & 0 \\
0 & 1 & 0
\end{array}\right)\left(\begin{array}{c}
\widehat{N}_{n+1} \\
\widehat{N}_{n} \\
\widehat{N}_{n-1}
\end{array}\right) .
$$

For $n \geq 0$, we define

$$
B_{n}=\left(\begin{array}{ccc}
\sum_{k=0}^{n+1} \widehat{N}_{k} & -5 \sum_{k=0}^{n} \widehat{N}_{k}+3 \sum_{k=0}^{n-1} \widehat{N}_{k} & 3 \sum_{k=0}^{n} \widehat{N}_{k} \\
\sum_{k=0}^{n} \widehat{N}_{k} & -5 \sum_{k=0}^{n-1} \widehat{N}_{k}+3 \sum_{k=0}^{n-2} \widehat{N}_{k} & 3 \sum_{k=0}^{n-1} \widehat{N}_{k} \\
\sum_{k=0}^{n-1} \widehat{N}_{k} & -5 \sum_{k=0}^{n-2} \widehat{N}_{k}+3 \sum_{k=0}^{n-3} \widehat{N}_{k} & 3 \sum_{k=0}^{n-2} \widehat{N}_{k}
\end{array}\right)
$$

and

$$
C_{n}=\left(\begin{array}{ccc}
b_{n+1} & -5 b_{n}+3 b_{n-1} & 3 b_{n} \\
b_{n} & -5 b_{n-1}+3 b_{n-2} & 3 b_{n-1} \\
b_{n-1} & -5 b_{n-2}+3 b_{n-3} & 3 b_{n-2}
\end{array}\right)
$$

By convention, we assume that

$$
\sum_{k=0}^{-1} \widehat{N}_{k}=0, \quad \sum_{k=0}^{-2} \widehat{N}_{k}=\frac{1}{3}, \quad \sum_{k=0}^{-3} \widehat{N}_{k}=\frac{5}{9} .
$$

Theorem 7.1. For all integers $m, n \geq 0$, we have

(a) $B_{n}=A^{n}$.

(b) $C_{1} A^{n}=A^{n} C_{1}$. 
(c) $C_{n+m}=C_{n} B_{m}=B_{m} C_{n}$.

Proof. (a) Proof can be done by mathematical induction on $n$.

(b) After matrix multiplication, (b) follows.

(c) We have

$$
\begin{aligned}
& A C_{n-1}=\left(\begin{array}{ccc}
4 & -5 & 3 \\
1 & 0 & 0 \\
0 & 1 & 0
\end{array}\right)\left(\begin{array}{ccc}
b_{n} & -5 b_{n-1}+3 b_{n-2} & 3 b_{n-1} \\
b_{n-1} & -5 b_{n-2}+3 b_{n-3} & 3 b_{n-2} \\
b_{n-2} & -5 b_{n-3}+3 b_{n-4} & 3 b_{n-3}
\end{array}\right) \\
& =\left(\begin{array}{ccc}
b_{n+1} & -5 b_{n}+3 b_{n-1} & 3 b_{n} \\
b_{n} & -5 b_{n-1}+3 b_{n-2} & 3 b_{n-1} \\
b_{n-1} & -5 b_{n-2}+3 b_{n-3} & 3 b_{n-2}
\end{array}\right)=C_{n},
\end{aligned}
$$

i.e., $C_{n}=A C_{n-1}$. From the last equation, using induction, we obtain $C_{n}=$ $A^{n-1} C_{1}$. Now

$$
C_{n+m}=A^{n+m-1} C_{1}=A^{n-1} A^{m} C_{1}=A^{n-1} C_{1} A^{m}=C_{n} B_{m}
$$

and similarly

$$
C_{n+m}=B_{m} C_{n}
$$

Some properties of matrix $A^{n}$ can be given as

$$
A^{n}=4 A^{n-1}-5 A^{n-2}+3 A^{n-3}=\frac{5}{3} A^{n+1}-\frac{4}{3} A^{n+2}+\frac{1}{3} A^{n+3}
$$

and

$$
A^{n+m}=A^{n} A^{m}=A^{m} A^{n}
$$

and

$$
\operatorname{det}\left(A^{n}\right)=3^{n}
$$

for all integers $m, n \geq 0$. 
Theorem 7.2. For $m, n \geq 0$, we have

$$
\begin{aligned}
b_{n+m} & =b_{n} \sum_{k=0}^{m+1} \widehat{N}_{k}+b_{n-1}\left(-5 \sum_{k=0}^{m} \widehat{N}_{k}+3 \sum_{k=0}^{m-1} \widehat{N}_{k}\right)+3 b_{n-2} \sum_{k=0}^{m} \widehat{N}_{k} \\
& =b_{n} \sum_{k=0}^{m+1} \widehat{N}_{k}+\left(-5 b_{n-1}+3 b_{n-2}\right) \sum_{k=0}^{m} \widehat{N}_{k}+3 b_{n-1} \sum_{k=0}^{m-1} \widehat{N}_{k} .
\end{aligned}
$$

Proof. From the equation $C_{n+m}=C_{n} B_{m}=B_{m} C_{n}$, we see that an element of $C_{n+m}$ is the product of row $C_{n}$ and a column $B_{m}$. From the last equation, we say that an element of $C_{n+m}$ is the product of a row $C_{n}$ and column $B_{m}$. We just compare the linear combination of the 2 nd row and 1st column entries of the matrices $C_{n+m}$ and $C_{n} B_{m}$. This completes the proof.

Corollary 7.3. For $m, n \geq 0$, we have

$$
\begin{aligned}
\widehat{N}_{n+m} & =\widehat{N}_{n} \sum_{k=0}^{m+1} \widehat{N}_{k}+\widehat{N}_{n-1}\left(-5 \sum_{k=0}^{m} \widehat{N}_{k}+3 \sum_{k=0}^{m-1} \widehat{N}_{k}\right)+3 \widehat{N}_{n-2} \sum_{k=0}^{m} \widehat{N}_{k}, \\
\widehat{U}_{n+m} & =\widehat{U}_{n} \sum_{k=0}^{m+1} \widehat{N}_{k}+\widehat{U}_{n-1}\left(-5 \sum_{k=0}^{m} \widehat{N}_{k}+3 \sum_{k=0}^{m-1} \widehat{N}_{k}\right)+3 \widehat{U}_{n-2} \sum_{k=0}^{m} \widehat{N}_{k}, \\
\widehat{H}_{n+m} & =\widehat{H}_{n} \sum_{k=0}^{m+1} \widehat{N}_{k}+\widehat{H}_{n-1}\left(-5 \sum_{k=0}^{m} \widehat{N}_{k}+3 \sum_{k=0}^{m-1} \widehat{N}_{k}\right)+3 \widehat{H}_{n-2} \sum_{k=0}^{m} \widehat{N}_{k},
\end{aligned}
$$

From Corollary 6.2, we know that for $n \geq 0$,

$$
\sum_{k=0}^{n} \widehat{N}_{k}=\widehat{N}_{n+3}-3 \widehat{N}_{n+2}+2 \widehat{N}_{n+1}
$$

So, Theorem 7.2 and Corollary 7.3 can be written in the following forms:

Theorem 7.4. For $m, n \geq 0$, we have

$$
\begin{aligned}
b_{n+m}= & \left(\widehat{N}_{m+4}-3 \widehat{N}_{m+3}+2 \widehat{N}_{m+2}\right) b_{n} \\
& +\left(-5 \widehat{N}_{m+3}+18 \widehat{N}_{m+2}-19 \widehat{N}_{m+1}+6 \widehat{N}_{m}\right) b_{n-1} \\
& +3\left(\widehat{N}_{m+3}-3 \widehat{N}_{m+2}+2 \widehat{N}_{m+1}\right) b_{n-2} .
\end{aligned}
$$


Remark 7.5. By induction, it can be proved that for all integers $m, n \leq 0,7.4$ holds. So, for all integers $m, n, 7.4$ is true.

Corollary 7.6. For all integers $m, n$, we have

$$
\begin{aligned}
N_{n+m}= & \left(\widehat{N}_{m+4}-3 \widehat{N}_{m+3}+2 \widehat{N}_{m+2}\right) N_{n} \\
& +\left(-5 \widehat{N}_{m+3}+18 \widehat{N}_{m+2}-19 \widehat{N}_{m+1}+6 \widehat{N}_{m}\right) N_{n-1} \\
& +3\left(\widehat{N}_{m+3}-3 \widehat{N}_{m+2}+2 \widehat{N}_{m+1}\right) N_{n-2} \\
U_{n+m}= & \left(\widehat{N}_{m+4}-3 \widehat{N}_{m+3}+2 \widehat{N}_{m+2}\right) U_{n} \\
& +\left(-5 \widehat{N}_{m+3}+18 \widehat{N}_{m+2}-19 \widehat{N}_{m+1}+6 \widehat{N}_{m}\right) U_{n-1} \\
& +3\left(\widehat{N}_{m+3}-3 \widehat{N}_{m+2}+2 \widehat{N}_{m+1}\right) U_{n-2}, \\
H_{n+m}= & \left(\widehat{N}_{m+4}-3 \widehat{N}_{m+3}+2 \widehat{N}_{m+2}\right) H_{n} \\
& +\left(-5 \widehat{N}_{m+3}+18 \widehat{N}_{m+2}-19 \widehat{N}_{m+1}+6 \widehat{N}_{m}\right) H_{n-1} \\
& +3\left(\widehat{N}_{m+3}-3 \widehat{N}_{m+2}+2 \widehat{N}_{m+1}\right) H_{n-2} .
\end{aligned}
$$

Now, we consider non-positive subscript cases. For $n \geq 0$, we define

$$
B_{-n}=\left(\begin{array}{ccc}
-\sum_{k=0}^{n-2} \widehat{N}_{-k} & \left.5 \sum_{k=0}^{n-1} \widehat{N}_{-k}-3 \sum_{k=0}^{n} \widehat{N}_{-k}\right) & -3 \sum_{k=0}^{n-1} \widehat{N}_{-k} \\
-\sum_{k=0}^{n-1} \widehat{N}_{-k} & \left.5 \sum_{k=0}^{n} \widehat{N}_{-k}-3 \sum_{k=0}^{n+1} \widehat{N}_{-k}\right) & -3 \sum_{k=0}^{n} \widehat{N}_{-k} \\
-\sum_{k=0}^{n} \widehat{N}_{-k} & \left.5 \sum_{k=0}^{n+1} \widehat{N}_{-k}-3 \sum_{k=0}^{n+2} \widehat{N}_{-k}\right) & -3 \sum_{k=0}^{n+1} \widehat{N}_{-k}
\end{array}\right)
$$

and

$$
C_{-n}=\left(\begin{array}{ccc}
b_{-n+1} & -5 b_{-n}+3 b_{-n-1} & 3 b_{-n} \\
b_{-n} & -5 b_{-n-1}+3 b_{-n-2} & 3 b_{-n-1} \\
b_{-n-1} & -5 b_{-n-2}+3 b_{-n-3} & 32 b_{-n-2}
\end{array}\right)
$$

By convention, we assume that

$$
\sum_{k=0}^{-1} \widehat{N}_{-k}=0, \quad \sum_{k=0}^{-2} \widehat{N}_{-k}=-1 .
$$

Theorem 7.7. For all integers $m, n \geq 0$, we have

(a) $B_{-n}=A^{-n}$.

(b) $C_{-1} A^{-n}=A^{-n} C_{-1}$. 
(c) $C_{-n-m}=C_{-n} B_{-m}=B_{-m} C_{-n}$.

Proof. (a) Proof can be done by mathematical induction on $n$.

(b) After matrix multiplication, (b) follows.

(c) We have

$$
\begin{aligned}
A^{-1} C_{-n-1} & =\left(\begin{array}{ccc}
4 & -5 & 3 \\
1 & 0 & 0 \\
0 & 1 & 0
\end{array}\right)\left(\begin{array}{ccc}
b_{-n} & -5 b_{-n-1}+3 b_{-n-2} & 3 b_{-n-1} \\
b_{-n-1} & -5 b_{-n-2}+3 b_{-n-3} & 3 b_{-n-2} \\
b_{-n-2} & -5 b_{-n-3}+3 b_{-n-4} & 3 b_{-n-3}
\end{array}\right) \\
& =\left(\begin{array}{ccc}
b_{-n+1} & -5 b_{-n}+3 b_{-n-1} & 3 b_{-n} \\
b_{-n} & -5 b_{-n-1}+3 b_{-n-2} & 3 b_{-n-1} \\
b_{-n-1} & -5 b_{-n-2}+3 b_{-n-3} & 3 b_{-n-2}
\end{array}\right)=C_{-n},
\end{aligned}
$$

i.e. $C_{-n}=A^{-1} C_{-n-1}$. From the last equation, using induction, we obtain $C_{-n}=A^{-n-1} C_{-1}$. Now,

$$
C_{-n-m}=A^{-n-m-1} C_{-1}=A^{-n-1} A^{-m} C_{-1}=A^{-n-1} C_{-1} A^{-m}=C_{-n} B_{-m}
$$

and similarly,

$$
C_{-n-m}=B_{-m} C_{-n}
$$

Some properties of matrix $A^{-n}$ can be given as

$$
A^{-n}=4 A^{-n-1}-5 A^{-n-2}+3 A^{-n-3}=\frac{5}{3} A^{-n+1}-\frac{4}{3} A^{-n+2}+\frac{1}{3} A^{-n+3}
$$

and

$$
A^{-n-m}=A^{-n} A^{-m}=A^{-m} A^{-n}
$$

and

$$
\operatorname{det}\left(A^{-n}\right)=3^{-n}
$$

for all integers $m, n \geq 0$. 
Theorem 7.8. For $m, n \geq 0$, we have

$$
\begin{aligned}
b_{-n-m} & =-b_{-n} \sum_{k=0}^{m-2} \widehat{N}_{-k}-b_{-n-1}\left(-5 \sum_{k=0}^{m-1} \widehat{N}_{-k}+3 \sum_{k=0}^{m} \widehat{N}_{-k}\right)-3 b_{-n-2} \sum_{k=0}^{m-1} \widehat{N}_{-k} \\
& =-b_{-n} \sum_{k=0}^{m-2} \widehat{N}_{-k}-\left(-5 b_{-n-1}+3 b_{-n-2}\right) \sum_{k=0}^{m-1} \widehat{N}_{-k}-3 b_{-n-1} \sum_{k=0}^{m} \widehat{N}_{-k} .
\end{aligned}
$$

Proof. From the equation $C_{-n-m}=C_{-n} B_{-m}=B_{-m} C_{-n}$, we see that an element of $C_{-n-m}$ is the product of row $C_{-n}$ and a column $B_{-m}$. From the last equation, we say that an element of $C_{-n-m}$ is the product of a row $C_{-n}$ and column $B_{-m}$. We just compare the linear combination of the 2nd row and 1st column entries of the matrices $C_{-n-m}$ and $C_{-n} B_{-m}$. This completes the proof.

Corollary 7.9. For $m, n \geq 0$, we have

$$
\begin{aligned}
\widehat{N}_{-n-m}= & -\widehat{N}_{-n} \sum_{k=0}^{m-2} \widehat{N}_{-k}-\widehat{N}_{-n-1}\left(-5 \sum_{k=0}^{m-1} \widehat{N}_{-k}+3 \sum_{k=0}^{m} \widehat{N}_{-k}\right) \\
& -3 \widehat{N}_{-n-2} \sum_{k=0}^{m-1} \widehat{N}_{-k}, \\
\widehat{U}_{-n-m}= & -\widehat{U}_{-n} \sum_{k=0}^{m-2} \widehat{N}_{-k}-\widehat{U}_{-n-1}\left(-5 \sum_{k=0}^{m-1} \widehat{N}_{-k}+3 \sum_{k=0}^{m} \widehat{N}_{-k}\right) \\
& -3 \widehat{U}_{-n-2} \sum_{k=0}^{m-1} \widehat{N}_{-k}, \\
\widehat{H}_{-n-m}= & -\widehat{H}_{-n} \sum_{k=0}^{m-2} \widehat{N}_{-k}-\widehat{H}_{-n-1}\left(-5 \sum_{k=0}^{m-1} \widehat{N}_{-k}+3 \sum_{k=0}^{m} \widehat{N}_{-k}\right) \\
& -3 \widehat{H}_{-n-2} \sum_{k=0}^{m-1} \widehat{N}_{-k} .
\end{aligned}
$$

From Corollary 6.6, we know that for $n \geq 1$,

$$
\sum_{k=1}^{n} \widehat{N}_{-k}=-2 \widehat{N}_{-n-1}+2 \widehat{N}_{-n-2}-3 \widehat{N}_{-n-3} .
$$


Since $\widehat{N}_{0}=0$, it follows that

$$
\sum_{k=0}^{n} \widehat{N}_{-k}=-2 \widehat{N}_{-n-1}+2 \widehat{N}_{-n-2}-3 \widehat{N}_{-n-3} .
$$

So, Theorem 7.8 and Corollary 7.8 can be written in the following forms.

Theorem 7.10. For $m, n \geq 0$, we have

$$
\begin{aligned}
b_{-n-m}= & \left(2 \widehat{N}_{-m+1}-2 \widehat{N}_{-m}+3 \widehat{N}_{-m-1}\right) b_{-n} \\
& +\left(-10 \widehat{N}_{-m}+16 \widehat{N}_{-m-1}-21 \widehat{N}_{-m-2}+9 \widehat{N}_{-m-3}\right) b_{-n-1} \\
& +3\left(2 \widehat{N}_{-m}-2 \widehat{N}_{-m-1}+3 \widehat{N}_{-m-2}\right) b_{-n-2} .
\end{aligned}
$$

Remark 7.11. By induction, it can be proved that for all integers $m, n \leq 0,7.5$ holds. So, for all integers $m, n, 7.5$ is true.

Corollary 7.12. For all integers $m, n$, we have

$$
\begin{aligned}
\widehat{N}_{-n-m}= & \left(2 \widehat{N}_{-m+1}-2 \widehat{N}_{-m}+3 \widehat{N}_{-m-1}\right) \widehat{N}_{-n} \\
& +\left(-10 \widehat{N}_{-m}+16 \widehat{N}_{-m-1}-21 \widehat{N}_{-m-2}+9 \widehat{N}_{-m-3}\right) \widehat{N}_{-n-1} \\
& +3\left(2 \widehat{N}_{-m}-2 \widehat{N}_{-m-1}+3 \widehat{N}_{-m-2}\right) \widehat{N}_{-n-2}, \\
\widehat{U}_{-n-m}= & \left(2 \widehat{N}_{-m+1}-2 \widehat{N}_{-m}+3 \widehat{N}_{-m-1}\right) \widehat{U}_{-n} \\
& +\left(-10 \widehat{N}_{-m}+16 \widehat{N}_{-m-1}-21 \widehat{N}_{-m-2}+9 \widehat{N}_{-m-3}\right) \widehat{U}_{-n-1} \\
& +3\left(2 \widehat{N}_{-m}-2 \widehat{N}_{-m-1}+3 \widehat{N}_{-m-2}\right) \widehat{U}_{-n-2}, \\
H_{-n-m}= & \left(2 \widehat{N}_{-m+1}-2 \widehat{N}_{-m}+3 \widehat{N}_{-m-1}\right) H_{-n} \\
& +\left(-10 \widehat{N}_{-m}+16 \widehat{N}_{-m-1}-21 \widehat{N}_{-m-2}+9 \widehat{N}_{-m-3}\right) H_{-n-1} \\
& +3\left(2 \widehat{N}_{-m}-2 \widehat{N}_{-m-1}+3 \widehat{N}_{-m-2}\right) H_{-n-2} .
\end{aligned}
$$

\section{References}

[1] P. Barry, On integer-sequence-based constructions of generalized Pascal triangles, Journal of Integer Sequences 9 (2006), Article 06.2.4.

[2] I. Bruce, A modified Tribonacci sequence, The Fibonacci Quarterly 22(3) (1984), 244-246. 
[3] M. Catalani, Identities for Tribonacci-related sequences, 2002. arXiv preprint: https://arxiv.org/pdf/math/0209179.pdf math/0209179

[4] E. Choi, Modular Tribonacci numbers by matrix method, J. Korean Soc. Math. Educ. Ser. B: Pure Appl. Math. 20(3) (2013), 207-221. https://doi.org/10.7468/jksmeb.2013.20.3.207

[5] M. Elia, Derived sequences, the Tribonacci recurrence and cubic forms, The Fibonacci Quarterly 39(2) (2001), 107-115.

[6] M. C. Er, Sums of Fibonacci numbers by matrix methods, Fibonacci Quart. 22(3) (1984), 204-207.

[7] H. W. Gould, Series transformations for finding recurrences for sequences, The Fibonacci Quarterly 28(2) (1990), 166-171.

[8] P. Haukkanen, Formal power series for binomial sums of sequences of numbers, The Fibonacci Quarterly 31(1) (1993), 28-31.

[9] A. F. Horadam, A generalized Fibonacci sequence, American Mathematical Monthly 68 (1961), 455-459. https://doi.org/10.1080/00029890.1961.11989696

[10] A. F. Horadam, Basic properties of a certain generalized sequence of numbers, Fibonacci Quarterly 3.3 (1965), 161-176.

[11] A. F. Horadam, Special properties of the sequence $w_{n}(a, b ; p, q)$, Fibonacci Quarterly $5(5)$ (1967), 424-434.

[12] A. F. Horadam, Generating functions for powers of a certain generalized sequence of numbers, Duke Math. J. 32 (1965), 437-446. https://doi.org/10.1215/S0012-7094-65-03244-8

[13] F.T. Howard and F. Saidak, Zhou's theory of constructing identities, Congress Numer. 200 (2010), 225-237.

[14] D. Kalman, Generalized Fibonacci numbers by matrix methods, Fibonacci Quart. 20(1) (1982), 73-76.

[15] D. E. Knuth, The Art of Computer Programming 3, Reading, MA: Addison Wesley, 1973. 
[16] P. Y. Lin, De Moivre-Type identities for the Tribonacci numbers, The Fibonacci Quarterly 26 (1988), 131-134.

[17] S. Pethe, Some identities for Tribonacci sequences, The Fibonacci Quarterly 26(2) (1988), 144-151.

[18] H. Prodinger, Some information about the Binomial transform, The Fibonacci Quarterly 32.5 (1994), 412-415.

[19] A. Scott, T. Delaney and V. Hoggatt, Jr., The Tribonacci sequence, The Fibonacci Quarterly 15(3) (1977), 193-200.

[20] N. J. A. Sloane, The On-line Encyclopedia of Integer Sequences, http://oeis.org/

[21] A. Shannon, Tribonacci numbers and Pascal's pyramid, The Fibonacci Quarterly 15(3) (1977), pp. 268 and 275.

[22] Y. Soykan, Simson identity of generalized m-step Fibonacci numbers, Int. J. Adv. Appl. Math. Mech. 7(2) (2019), 45-56.

[23] Y. Soykan, Tribonacci and Tribonacci-Lucas sedenions, Mathematics 7(1) (2019), 74. https://doi.org/10.3390/math7010074

[24] Y. Soykan, Summing formulas for generalized Tribonacci numbers, Universal Journal of Mathematics and Applications 3(1) (2020), 1-11.

https://doi.org/10.32323/ujma.637876

[25] Y. Soykan, Generalized Tribonacci numbers: summing formulas, Int. J. Adv. Appl. Math. Mech. 7(3) (2020), 57-76.

[26] Y. Soykan, A closed formula for the sums of squares of generalized Tribonacci numbers, Journal of Progressive Research in Mathematics 16(2) (2020), 2932-2941.

[27] Y. Soykan, On the sums of squares of generalized Tribonacci numbers: closed formulas of $\sum_{k=0}^{n} x^{k} W_{k}^{2}$, Archives of Current Research International 20(4) (2020), 22-47. https://doi.org/10.9734/acri/2020/v20i430187

[28] Y. Soykan, On generalized Narayana numbers, Int. J. Adv. Appl. Math. Mech. 7(3) (2020), 43-56.

[29] W. Spickerman, Binet's formula for the Tribonacci sequence, The Fibonacci Quarterly 20 (1982), 118-120. 
[30] M. Z. Spivey, Combinatorial sums and finite differences, Discrete Math. 307 (2007), 3130-3146. https://doi.org/10.1016/j.disc.2007.03.052

[31] C. C. Yalavigi, Properties of Tribonacci numbers, The Fibonacci Quarterly 10(3) (1972), 231-246.

This is an open access article distributed under the terms of the Creative Commons Attribution License (http://creativecommons.org/licenses/by/4.0/), which permits unrestricted, use, distribution and reproduction in any medium, or format for any purpose, even commercially provided the work is properly cited. 